\title{
Revisiting distinctive processes in memory
}

\author{
MICHAEL J. CORTESE \\ College of Charleston, Charleston, South Carolina \\ JASON M. WATSON \\ University of Utah, Salt Lake City, Utah \\ MAYA M. KHANNA \\ University of Michigan, Ann Arbor, Michigan \\ and \\ MATHIE MCCALLION \\ College of Charleston, Charleston, South Carolina
}

\begin{abstract}
In three experiments, we examined the relationship between orthographic and phonological distinctiveness and incidental recall. In each experiment, participants were given a surprise free recall test after they read words aloud as quickly and accurately as possible. The pattern of results replicated those reported in Cortese, Watson, Wang, and Fugett (2004) for intentional and explicit free recall and recognition memory tasks in which items were read silently. Specifically, we found that phonological-to-orthographic neighborhood size influenced recall performance, whereas orthographic-to-phonological consistency and phonological-to-orthographic consistency did not. Also, we failed to replicate the orthographic-tophonological consistency effect reported by Hirshman and Jackson (1997), and argue that their results were due to a confounding of consistency with phonological neighborhood size. Our results suggest that the processing of words sharing both orthography and phonology with a large number of words produces interference that reduces one's ability to remember them.
\end{abstract}

Relating distinctive properties of objects to memory for these objects has interested cognitive psychologists for decades. In typical studies that have examined distinctiveness, stimuli are usually made distinct in some way. For example, a word in a list may be made distinct by underlining it, presenting it in a different color, and so forth. The von Restorff effect refers to this better memory performance for distinct items (see, e.g., Wallace, 1965).

Interestingly, words contain orthographic and phonological characteristics that may render them distinct (or not) due to the relationship that they have with other words. Moreover, this distinctiveness may influence memory. For example, Hunt and Elliott (1980) reported that orthographically distinctive words (e.g., phlegm) were recalled at a higher rate than were orthographically indistinct words (e.g., primate). Also, Hirshman and Jackson (1997) reported that words with inconsistent orthographic-to-phonological correspondences (e.g., plaid) were recalled at a higher rate than were words with consistent orthographic-to-phonological correspondences. More recently, Cortese and colleagues (Cortese, Watson, Wang, \& Fugett, 2004) reported that words with few phonological-to-orthographic neighbors (e.g., pulp) were recalled and recognized at a higher rate

Correspondence for this article should be addressed to M. J. Cortese, Department of Psychology, University of Nebraska, 6001 Dodge St., Omaha, NE 68182 (e-mail: mcortese@mail.unomaha.edu). than were words with many phonological-to-orthographic neighbors (e.g., tuck). The number of phonological-toorthographic neighbors reflects the number of other words that share both the orthographic and phonological rime with a target word. For example, the neighbors of tuck are duck, luck, puck, truck, stuck, and so forth. In addition to phonological-to-orthographic neighborhood size, Cortese et al. examined orthographic-to-phonological consistency and phonological-to-orthographic consistency, but neither variable produced a significant effect.

Cortese and colleagues related their findings to contemporary models of word processing (e.g., Coltheart, Rastle, Perry, Langdon, \& Ziegler, 2001; Plaut, McClelland, Seidenberg, \& Patterson, 1996) and an interference framework. Specifically, when one encodes and/or retrieves a target word, the representations associated with other similar words become activated, and this activation interferes with memory for the target word. Words sharing characteristics with many other words produce more interference than do words sharing characteristics with a smaller number of words.

The factors examined in the present study across three experiments are identical to those examined by Cortese et al. (2004) and include (1) the number of phonological-to-orthographic neighbors (Experiment 1A), (2) phonological-to-orthographic consistency (Experiment 1B), and (3) orthographic-to-phonological consistency (Experiment 1C). Phonological-to-orthographic 
consistency refers to the degree to which words sharing a phonological rime with a target word are spelled similarly. For example, tuck is consistent because all of its phonological neighbors contain the same spelling. In contrast, hype is inconsistent because many of its phonological neighbors (e.g., ripe, gripe, pipe, etc.) do not share the same spelling, although they do share the same sound (e.g., /arp/). Orthographic-to-phonological consistency refers to the degree to which similarly spelled words have the same pronunciation (see, e.g., Cortese \& Simpson, 2000). For example, pulp is consistent, because the letters it contains are associated most strongly with the phonemes found in pulp. In contrast, plaid is inconsistent, because aid is not usually pronounced /æd/ (e.g., maid, raid, paid, said, etc.).

We use the terms orthographic-to-phonological consistent (OPC) and inconsistent (OPI) to indicate the orthographic-to-phonological consistency of items. Similarly, the terms phonological-to-orthographic consistent (POC) and inconsistent (POI) are used to indicate the phonological-to-orthographic consistency of the items. Consistent words on both OP and PO dimensions with few rhyming neighbors are denoted as low $N$, and words with many rhyming neighbors are denoted as high $N$. The four types of words used in the present study are

(1) OPC and POC: low $N$ (e.g., pulp)

(2) OPC and POC: high $N$ (e.g., tuck)

(3) OPC and POI: low $N$ (e.g., hype)

(4) OPI and POI: low $N$ (e.g., plaid)

The four conditions of words employed in the present study allow for three separate comparisons:

(A) The number of PO neighbors (Conditions 1 vs. 2)

(B) PO consistency (Conditions 1 vs. 3)

(C) OP consistency (Conditions 3 vs. 4)

Each comparison was conducted as a separate experiment. Frequency and length were equated across the different conditions of words. Conditions 1 and 2 differed significantly in terms of orthographic neighborhood size (Coltheart, Davelaar, Jonasson, \& Besner, 1977) so that variable was controlled statistically via analysis of covariance in items analyses that involved these two conditions. Otherwise, the conditions did not differ in terms of orthographic neighborhood size.

It is important to note that the (lack of a) orthographicto-phonological consistency effect reported by Cortese et al. (2004) is at odds with the effect reported by Hirshman and Jackson (1997). For this reason, the main purpose of the present study was to resolve the apparent discrepancy between the results originally reported by Hirshman and Jackson (1997) and those recently reported by Cortese et al. As we see it, there were three possible reasons for the discrepant results. First, Hirshman and Jackson employed an incidental learning task, and Cortese and colleagues employed an intentional learning procedure. In other words, Hirshman and Jackson's participants did not know that their memory would later be tested when they encoded target words, whereas Cortese et al.'s participants did. Although there is no evidence that suggests that incidental versus intentional learning will differentially affect consistency, it is possible that incidental learning is more sensitive to this manipulation. Second, Hirshman and Jackson's participants encoded target words by reading them aloud, and Cortese et al.'s participants encoded target words by reading them silently. Research on reading has demonstrated that effects of orthographic-to-phonological consistency are stronger in reading aloud tasks than in silent reading tasks (e.g., the lexical decision task; Balota, Cortese, Sergent-Marshall, Spieler, \& Yap, 2004; Cortese, 1998). Thus, the emphasis on orthographic-to-phonological processes in reading aloud may produce an effect of orthographic-to-phonological consistency because attention is focused on the very process that distinguishes stimuli. Third, in the Hirshman and Jackson (1997) study, orthographic-to-phonological consistency was confounded with phonological-to-orthographic neighborhood size, and in the Cortese et al. (2004) study, these factors were not confounded. Therefore, it is possible that Hirshman and Jackson's results should not be attributed to consistency but to neighborhood size instead.

In the present study, we employed the stimuli (all of which were monosyllabic words) of Cortese et al. (2004) and the procedure of Hirshman and Jackson (1997). Therefore, if the procedural differences were responsible for the discrepant results between studies, then an orthographicto-phonological consistency effect should emerge in the present study. However, if the effect reported by Hirshman and Jackson was due to a confounding of orthographic-tophonological consistency and phonological-to-orthographic neighborhood size, then we should find results comparable to those reported by Cortese et al.

\section{EXPERIMENT 1A PO Neighborhood Size}

In Experiment 1A, OP and PO consistency were held constant, and the number of $\mathrm{PO}$ neighbors varied between conditions. Even though the procedure of the present study differed from that of Cortese et al. (2004), we still predicted that POC low- $N$ words would be recalled at a higher rate than would POC high- $N$ words. Specifically, if phonological-to-orthographic representations are activated during encoding and/or retrieval, then low- $N$ words should produce less interference from their neighbors than high- $N$ words.

\section{Method}

\section{Participants}

Thirty-one College of Charleston undergraduates participated in Experiment 1A.

\section{Materials}

The items are from Cortese et al. (2004; see their Appendix for the list of words). Stimulus characteristics for the words used in the three experiments are presented in Table 1. The items consisted of 
Table 1

Stimulus Characteristics for the Words Used in Experiments 1A-1C

\begin{tabular}{lccccc}
\hline & Frequency & Letter Length & No. of Phonemes & PO Friends & PO Enemies \\
\hline OPC and POC-Low $N$ & 5.6 & 4.5 & 3.86 & 0.5 & 0.0 \\
OPC and POC-High $N$ & 6.3 & 4.5 & 4.07 & 10.7 & 0.0 \\
OPC and POI-Low $N$ & 7.3 & 4.3 & 3.43 & 0.3 & 12.4 \\
OPI and POI-Low $N$ & 6.0 & 4.6 & 3.29 & 0.4 & 12.6 \\
\hline
\end{tabular}

Note-O, orthographic; P, phonological; C, consistent; I, inconsistent. Low $N$, few PO neighbors; High $N$, many PO neighbors. $P O$ friends refer to the number of words that contain the same phonological rime and spelling for that rime, and $P O$ enemies refer to the number of words that contain the same phonological rime and a different spelling for that rime.

28 monosyllabic words of low frequency (Kučera \& Francis, 1967). Fourteen words from each of two categories were selected: (1) OPC and POC low $N$ (e.g., pulp), and (2) OPC and POC high $N$ (e.g., tuck). The 28 items used in Experiment 1A were part of an overall set of four categories of 14 items each ( 56 total items) that were used across the three experiments. Among (both $F \mathrm{~s}<1$ ) the four categories and between (all $p \mathrm{~s}>.26$ ) the experimental conditions, items were equated for frequency and orthographic length. In addition, no two words contained the same orthographic and phonological rime. $N$ (i.e., $P O$ friends in Table 1) refers to the number of words that contain both the same phonological rime and the same orthographic rime (Ziegler, Stone, \& Jacobs, 1997). In Table 1, PO enemies refers to the number of words that contain the same phonological rime and a different orthographic rime (Ziegler et al., 1997). No two words contained both the same orthographic and the same phonological rime. However, hurl and pearl as well as womb and plume contained the same phonological rimes, but neither pair contained the same orthographic rime. With these exceptions, no two words contained the same phonological rime, and no two words contained the same orthographic rime. Uppercase letters were used in all experiments in order to eliminate orthographic distinctiveness as a potential contributing factor (see Hunt \& Elliott, 1980). A practice list of 10 words was also constructed for each experiment. Practice trials were employed so that participants could familiarize themselves with the reading aloud task.

\section{Procedure}

During the reading aloud task, words were presented one at a time in uppercase letters in the center of the computer screen. The order of items was randomized for each participant. Each word appeared until the participant named it. ${ }^{1}$ The experimenter then coded the response as correct, incorrect, or noise (if the microphone failed to pick up the voice or some extraneous noise triggered the microphone). Participants were informed that they would be presented with a list of words, one at a time, and that they should read each word aloud as quickly and accurately as possible. They were not told about the memory test. Ten practice trials preceded the experiment. Immediately after the last word was read aloud, a message appeared on the monitor that instructed the participant to recall, in any order, as many of the words from the experiment as (s)he could by writing the words on a recall sheet. In addition, the message instructed the participant to be accurate in his/her responses. Across the three experiments, participants averaged 1.0 intrusions per list. The experimenter then provided the participant with the recall sheet.

\section{Design}

PO neighborhood size (low, high) was manipulated within participants. Proportion recalled and naming latency served as the dependent measures.

\section{Results and Discussion}

In all three experiments, data from responses coded as noise, and responses faster than $250 \mathrm{msec}$ and greater than
$1,000 \mathrm{msec}$, were removed from the analyses. This accounted for .02 of the responses in Experiment 1A, .03 of the responses in Experiment 1B, and .05 of the responses in Experiment 1C. Table 2 presents the mean naming latencies and proportion of errors for each condition for each experiment. Table 3 presents the mean proportion of recall for each condition of the experiment. Separate subject $\left(t_{\mathrm{s}}\right)$ and item analyses $\left(t_{\mathrm{i}}\right.$ or $F_{\mathrm{i}}$; Clark, 1973) are reported. ${ }^{2}$ For all the following analyses, all effects are significant at $p<$ .05 , unless noted. Note that $t<1$ is used to indicate that the absolute value of $t<1$.

\section{Latency and Error Data}

In Experiment $1 \mathrm{~A}$, low- $N$ words were named more quickly than high- $N$ words $\left[t_{\mathrm{s}}(30)=4.69, S E M=4.59\right.$; $\left.F_{\mathrm{i}}(1,25)=12.88, M S_{\mathrm{e}}=1,086.08\right]$. However, more errors were produced for low- $N$ words than for high- $N$ words $\left[t_{\mathrm{s}}(30)=2.28, S E M=.016 ; F_{\mathrm{i}}(1,25)=3.45, p<.08\right.$, $\left.M S_{\mathrm{e}}=.002\right]$.

\section{Recall Data}

Participants recalled a higher proportion of low- $N$ words than of high- $N$ words $\left[t_{\mathrm{s}}(30)=4.28, S E M=.026\right.$; $\left.F_{\mathrm{i}}(1,25)=10.30, M S_{\mathrm{e}}=.011\right]$.

\section{EXPERIMENT 1B PO Consistency}

In Experiment 1B, the number of $\mathrm{PO}$ neighbors and OP consistency were held constant, and PO consistency

Table 2

Reading Aloud Latencies and Proportion of Errors for Experiments 1A-1C

\begin{tabular}{crc}
\hline Measure & Latency & Prop. of Errors \\
\hline \multicolumn{3}{c}{ Experiment 1A } \\
OP Neighborhood Size & 578.6 & .05 \\
Low $N$ & 600.2 & .01 \\
High $N$ & Experiment 1B & \\
& & \\
PO Consistency & 615.0 & .03 \\
Consistent & 643.0 & .09 \\
Inconsistent & Experiment 1C & \\
OP Consistency & 706.0 & .06 \\
Consistent & 698.0 & .20 \\
Inconsistent &
\end{tabular}


Table 3

Proportion Recalled for Experiments 1A-1C

Experiment $1 \mathrm{~A}$

\begin{tabular}{lc}
\hline OP Neighborhood Size & Proportion Recalled \\
\hline Low $N$ & .28 \\
High $N$ & .17 \\
& Experiment 1B \\
\hline PO Consistency & \multicolumn{2}{c}{ Proportion Recalled } \\
\hline Consistent & .23 \\
Inconsistent & .27 \\
& Experiment 1C \\
\hline OP Consistency & Proportion Recalled \\
\hline Consistent & \multicolumn{2}{c}{.23} \\
Inconsistent & \multicolumn{2}{c}{}
\end{tabular}

varied between conditions. Although we had not found an effect of PO consistency previously, the emphasis of phonological processes in the reading aloud task might produce an effect of this variable. The literature suggested that $\mathrm{PO}$ consistency might affect reading aloud processes, but there was no clear consensus (see, e.g., Balota et al., 2004).

\section{Method}

\section{Participants}

Thirty-one College of Charleston undergraduates participated in Experiment 1B.

\section{Materials}

The items consisted of 28 monosyllabic words of low frequency (Kučera \& Francis, 1967). Fourteen words from each of two categories were selected: (1) OPC and POC low $N$ (e.g., pulp), and (2) OPC and POI low $N$ (e.g., hype). PO consistency (i.e., the number of PO friends and enemies in Table 1) was calculated according to the Ziegler et al. (1997) norms.

\section{Design}

PO consistency (consistent, inconsistent) was manipulated within participants. Proportion recalled and naming latency served as the dependent measures.

\section{Results and Discussion \\ Latency and Error Data}

Latency data from 1 participant were lost because of machine failure. In Experiment 1B, PO consistent words were named more quickly than $\mathrm{PO}$ inconsistent words $\left[t_{\mathrm{s}}(29)=3.47, S E M=8.07 ; t_{\mathrm{i}}(26)=1.80, S E M=11.79\right.$; $p<.09]$. In addition, a higher proportion of errors were committed in the naming of PO inconsistent words than in the naming of $\mathrm{PO}$ consistent words, but this effect was significant by subjects only $\left[t_{\mathrm{s}}(29)=4.21, S E M=.014\right.$; $\left.t_{\mathrm{i}}<1\right]$.

\section{Recall Data}

Although the proportion of recall was slightly higher for PO inconsistent words than for PO consistent words, this effect was not significant $\left[t_{\mathrm{s}}(30)=1.56, p>.12\right.$; $\left.t_{\mathrm{i}}<1\right]$.

\section{EXPERIMENT 1C OP Consistency}

In Experiment 1C, the number of $\mathrm{PO}$ neighbors and $\mathrm{PO}$ consistency were held constant, and OP consistency (e.g., Hirshman \& Jackson, 1997) varied between conditions. This experiment provided a straightforward examination of OP consistency without confounding this variable with PO neighborhood size. As previously stated, the emphasis of spelling-to-sound conversion in the reading aloud task may produce an effect of consistency. Hirshman and Jackson (1997) argued that the OP consistency effect arises during retrieval operations. Specifically, they stated that OP representations become activated during retrieval and serve as cues. Inconsistent words are facilitated more by this process than are consistent words, because of their more distinct OP mappings. If so, then we should replicate their results.

\section{Method}

\section{Materials}

Fourteen words from each of two categories were selected: (1) OPC and POI low $N$ (e.g., hype), and (2) OPI and POI low $N$ (e.g., plaid). OP consistency was also calculated according to the Ziegler et al. (1997) norms and/or the grapheme-to-phoneme correspondence rules of the Coltheart et al. (2001) model. Some words are classified as consistent by the Ziegler et al. (1997) norms because they do not contain any orthographic rime neighbors (e.g., yacht, niche, gauge, tongue, ache, and crepe). However, these words were considered inconsistent for the present study because they are highly inconsistent at the grapheme-to-phoneme level (see Berndt, Reggia, \& Mitchum, 1987). These words were submitted to the grapheme-to-phoneme correspondence rule translation procedure of Coltheart et al. (2001) at www.maccs.mq.edu.au/Utilities/regcelex .html. This procedure assigns pronunciation codes for words on the basis of the most common grapheme-to-phoneme mappings found in monosyllabic words. Indeed, all six words were given incorrect pronunciation codes.

\section{Design}

OP consistency (consistent, inconsistent) was manipulated within participants. Proportion recalled and naming latency served as the dependent measures.

\section{Participants}

Thirty-one College of Charleston undergraduates participated in Experiment 1C.

\section{Results and Discussion}

\section{Latency and Error Data}

Latency data from 1 participant were lost because of machine failure. Naming latencies to OPC consistent words were not significantly different from those to OPI words (both $t \mathrm{~s}<1$ ). However, a higher proportion of errors were committed in the pronunciation of OPI words than in the pronunciation of OPC words $\left[t_{\mathrm{s}}(29)=5.95\right.$, $\left.S E M=.023 ; t_{\mathrm{i}}(26)=1.86, p<.08, S E M=.050\right]$.

\section{Recall Data}

Although the proportion of recall was slightly higher for OPI words than for OPC words, this difference was not significant $\left[t_{\mathrm{s}}(30)=1.10, p>.28 ; \mathrm{t}_{\mathrm{i}}<1\right]$. 


\section{GENERAL DISCUSSION}

The overall pattern of memory data reported here is quite similar to that reported by Cortese et al. (2004). Across the two studies, four independent experiments have demonstrated that words sharing the orthography and phonology with many other words are associated with poor memory performance, whereas words sharing their orthography and phonology with few words are associated with good memory performance. Our hypothesis is that orthographic and phonological overlap among words produces interference that is greater for high- $N$ words than for low- $N$ words.

Therefore, we hypothesize that interference may arise during both encoding and retrieval. However, we note that in Cortese et al. (2004), the neighborhood size effect was obtained in both recognition and recall memory tasks. Since recognition emphasizes retrieval processes less than recall does, encoding seems to be implicated as a basis for the effect. In parallel-distributed-processing models (see, e.g., Seidenberg \& McClelland, 1989), high- $N$ words should activate more semantic features than should low$N$ words. For example, $u c k-/ \mathrm{uk} /$ is related to the meanings associated with tuck, luck, suck, buck, and so forth, whereas $u l p-/ \mathrm{ulp} /$ is associated with the meanings only of gulp and pulp. Therefore, a diffusion of semantic activation may occur for high- $N$ words that is the result of orthographic and phonological input patterns.

The primary focus of the present study was to resolve the discrepancy between the results reported by Cortese et al. (2004) and those reported by Hirshman and Jackson (1997). Specifically, in our previous study, we did not find an OP consistency effect in recall, whereas Hirshman and Jackson did. As previously discussed, our results could have differed from Hirshman and Jackson's either because of methodological factors or because of a confounding between PO neighborhood size and OP consistency in the Hirshman and Jackson study. In the present study, we employed the method of Hirshman and Jackson, we decoupled PO neighborhood size from OP consistency, and we found a clear effect of PO neighborhood size and no evidence for an OP consistency effect.

One might be concerned about the lack of an OP consistency effect in the latency data. However, in our comparison, we equated $\mathrm{PO}$ consistency effects by using only POI items. We also did not control for initial phoneme characteristics. We note that many standard reading aloud studies that have reported OP consistency effects have confounded OP and PO consistency. As Cortese et al. (2004) have noted, the inconsistent words in the Hirshman and Jackson (1997) study had fewer PO neighbors $(M=$ 1.3) than did the consistent words $(M=11.4)[t(24)=$ $5.90, p<.01]$. Interestingly, if we compare the two conditions that represent the types of items used by Hirshman and Jackson (high- $N$ OP consistent words - i.e., Condition 2; and OP inconsistent words-i.e., Condition 4), we replicate their results nicely. First, as assessed by an independent samples $t$ test, high- $N$ OP consistent words were named more quickly than low- $N$ OPI words $\left[t_{\mathrm{s}}(59)=\right.$ $\left.3.64, S E M=18.95 ; t_{\mathrm{i}}(26)=4.33, S E M=14.05\right]$. Also, fewer errors were committed to high- $N$ words than to OPI words $\left[t_{\mathrm{s}}(59)=10.32, S E M=.012 ; t_{\mathrm{i}}(26)=2.92\right.$, $S E M=.037]$. Finally, OPI words were recalled at a higher rate than were high- $N$ words $\left[t_{\mathrm{s}}(60)=4.22, S E M=.019\right.$; $\left.t_{\mathrm{i}}(26)=2.78, S E M=.026\right] .^{3}$

Finally, we note that in all the experiments, the condition associated with the higher proportion of mispronunciations also yielded the higher recall rate. However, it is unlikely that recall performance can be attributed to pronunciation difficulty per se. If difficulty of pronunciation were responsible for the recall performance, we would expect that OP inconsistent words would yield a significantly higher recall rate than would OP consistent words, but this did not occur. In addition, we performed a separate analysis on Experiment $1 \mathrm{~A}$ including the pronunciation accuracy rate as an additional covariate, and the $\mathrm{PO}$ neighborhood size effect remained significant $[F(1,24)=$ $\left.6.45, M S_{\mathrm{e}}=.011\right]$. In contrast, accuracy rate did not significantly predict recall performance $[F(1,24)=2.64$, $\left.p>.11, M S_{\mathrm{e}}=.011\right]$.

In summary, we employed a methodology very similar to that used by Hirshman and Jackson (1997), and found that PO neighborhood consistency influenced recall performance whereas OP consistency did not. This finding replicates the pattern reported by Cortese et al. (2004) and suggests that orthographic and phonological overlap among words is a source of memory interference.

\section{REFERENCES}

Balota, D. A., Cortese, M. J., Sergent-Marshall, S. D., Spieler, D. H., \& YAP, M. J. (2004). Visual word recognition for single syllable words. Journal of Experimental Psychology: General, 133, 283-316.

Berndt, R. S., Reggia, J. A., \& Mitchum, C. C. (1987). Empirically derived probabilities for grapheme-to-phoneme correspondences in English. Behavior Research Methods, Instruments, \& Computers, 19, 1-9.

Clark, H. (1973). The language-as-fixed-effect fallacy: A critique of language statistics in psychological research. Journal of Verbal Learning \& Verbal Behavior, 12, 335-339.

Coltheart, M., Davelaar, E., Jonasson, J. T., \& Besner, D. (1977). Access to the internal lexicon. In S. Dornic (Ed.), Attention and performance VI (pp. 535-555). Hillsdale, NJ: Erlbaum.

Coltheart, M., Rastle, K., Perry, C., Langdon, R., \& Ziegler, J. [C.] (2001). DRC: A dual route cascaded model of visual word recognition and reading aloud. Psychological Review, 108, 204-256.

Cortese, M. J. (1998). Revisiting serial position effects in reading. Journal of Memory \& Language, 39, 652-665.

Cortese, M. J., \& Simpson, G. B. (2000). Regularity effects in word naming: What are they? Memory \& Cognition, 28, 1269-1276.

Cortese, M. J., Watson, J. M., WAng, J., \& Fugett, A. (2004). Relating distinctive orthographic and phonological processes to episodic memory performance. Memory \& Cognition, 32, 632-639.

Hirshman, E., \& JACKSON, E. (1997). Distinctive perceptual processing memory. Journal of Memory \& Language, 36, 2-12.

Hunt, R. R., \& Elliott, J. M. (1980). The role of nonsemantic information in memory: Orthographic distinctiveness effects on retention. Journal of Experimental Psychology: General, 109, 49-74.

KuČERA, H., \& FrANCIS, W. N. (1967). Computational analysis of presentday American English. Providence, RI: Brown University Press.

Lupker, S. J., Brown, P., \& Colombo, L. (1997). Strategic control in 
a naming task: Changing routes or changing deadlines? Journal of Experimental Psychology: Learning, Memory, \& Cognition, 23, 570590.

Plaut, D. E., McClelland, J. L., Seidenberg, M. S., \& Patterson, K. E. (1996). Understanding normal and impaired reading: Computational principles in quasi-regular domains. Psychological Review, 103, 56-115.

Seidenberg, M. S., \& McClelland, J. L. (1989). A distributed, developmental model of word recognition and naming. Psychological Review, 96, 523-568.

WALLACE, W. P. (1965). Review of historical, empirical, and theoretical status of the von Restorff phenomenon. Psychological Bulletin, 63, 410-424.

Ziegler, J. C., Stone, G. O., \& Jacobs, A. M. (1997). What is the pronunciation for -ough and the spelling for $/ \mathrm{u} /$ ? A database for computing feedforward and feedback consistency in English. Behavior Research Methods, Instruments, \& Computers, 29, 600-618.

\section{NOTES}

1. The termination of items when the voice key was triggered differs slightly from the procedure employed by Hirshman and Jackson (1997). In their study, each word remained on the screen for approximately $1 \mathrm{sec}$. We note that the pattern of results reported here cannot be attributable to processing duration, because our low- $N$ items were named, on average, more quickly than other types of words.

2. An ANOVA was used to analyze items in Experiment 1 A because orthographic neighborhood size was included as a covariate. Orthographic neighborhood size did not differ reliably in any of the other comparisons, so $t$ tests were employed to analyze items in Experiments $1 \mathrm{~B}$ and $1 \mathrm{C}$.

3. Another interesting aspect of the latency data is that they conform quite nicely to the deadline hypothesis (Lupker, Brown, \& Colombo, 1997). According to this hypothesis, participants become sensitive to the average difficulty of the items in a list and respond accordingly. Specifically, they speed up their responses to the difficult items and slow down their responses to the easy items. In Experiment 1A, the items were not difficult to name because all items were both OP and PO consistent. In this situation, low- $N$ words were named in $579 \mathrm{msec}$. However, when the same words were named with more difficult items (Experiment 1B), the average latency was $615 \mathrm{msec}$ for the same low- $N$ words. Interestingly, POI words in Experiment 1B appeared with less difficult words in that experiment, and they were responded to in $643 \mathrm{msec}$, whereas in Experiment $1 \mathrm{C}$, they appeared with more difficult words, and the average latency for the same set of words increased to $707 \mathrm{msec}$.

(Manuscript received May 4, 2005; revision accepted for publication October 11, 2005.) 